\title{
Editorial
}

\section{Publishing with impact is the key to our success}

Cowin A

For referencing Cowin A. Publishing with impact is the key to our success. Wound Practice and Research 2020; $28(3): 105$.

DOI https://doi.org/10.33235/wpr.28.3.105

Publishing a research project, review or case study in an accredited peer reviewed journal such as Wound Practice and Research is a significant achievement and one that holds benefits for both the researcher and the organisation that hosts the journal eg Wounds Australia. It is only through publication that the research, including its scientific and practical contributions, is disseminated to others who are interested in the field.

Publishing enables researchers and practitioners to become aware of new knowledge and it helps to advance both the development of this knowledge as well as its application into clinical practice. The quality and impact of a journal is identified through how widely it is read, how often it is cited and its perception and image in the community. The higher the quality of the journal, the more difficult it can be to get articles accepted for publication. However, if successful it shows expertise in a field and an ability to conduct scientifically rigorous research. As the quality of the journal increases this further reflects on the stature of the institution hosting the publication.

While there are different recognition factors for each journal, which can be specific to a field or applicable across multiple disciplines, inclusion in reputable indexing agencies and databases and the attainment of an impact factor are

Prof Allison Cowin

Editor Wound Practice and Research considered critical when assessing journal quality and impact. As a measure of the growing impact of our journal, we are pleased to announce that Wound Practice and Research has been accepted for indexing by Clarivate Web of Science in its Emerging Sources Citation Index (ESCI). Journals accepted for coverage in this index must meet the following criteria: be peer reviewed, follow ethical publishing practices, meet specific technical requirements, have English language bibliographic information and be recommended or requested by a scholarly audience of Web of Science users. Journals that are indexed in the ESCI are discoverable on the Web of Science with full citation counts, author information and other enrichments. Articles in ESCI indexed journals are also included in an author's $h$-index calculation.

While it can take several years to generate an official impact factor, as they are calculated over the previous two years, we have been able to calculate the impact factor for Wound Practice and Research for 2019 to be 1.43. We are delighted with this starting point which reflects the quality of the papers published in our journal. Our next goal is to be included in the prestigious Medline database and we will keep you informed of our progress.

In the meantime, we are keen to see the journal continue to increase its international profile and impact and look forward to receiving many more submissions of your quality wound research, reviews and case reports that we know from our feedback are highly regarded by members of our wound community.

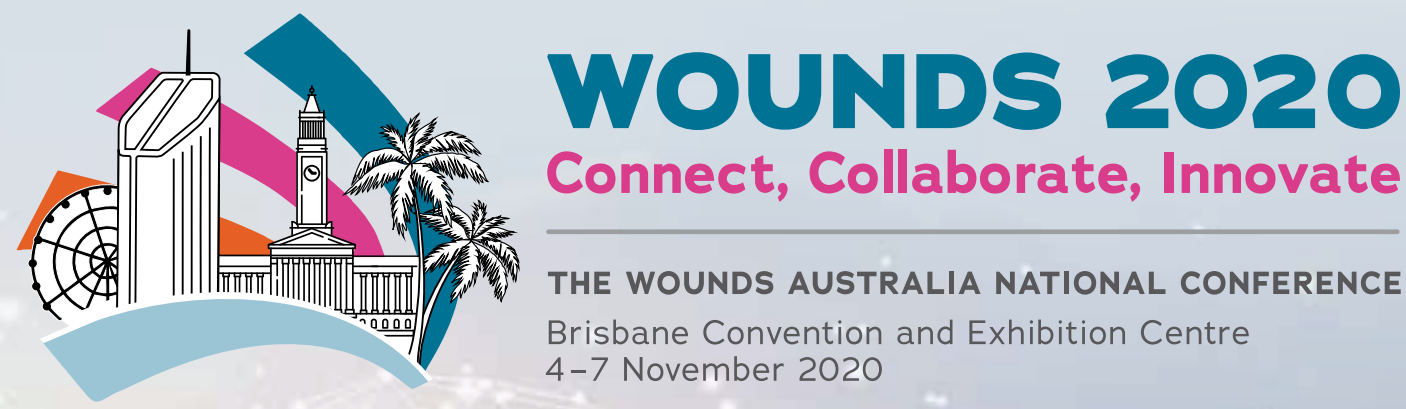

